Partial Differential Equations/Probability Theory

\title{
Asymptotic behavior of solutions of stochastic evolution equations for second grade fluids
}

\section{Comportement asymptotique des solutions d'équations d'évolution stochastiques des fluides de grade deux}

\author{
Paul André Razafimandimby, Mamadou Sango \\ Department of Mathematics and Applied Mathematics, University of Pretoria, Pretoria 0002, South Africa
}

\section{A R T I C L E I N F O}

\section{Article history:}

Received 10 January 2010

Accepted after revision 27 April 2010

Available online $\mathrm{xxxx}$

Presented by Pierre-Louis Lions

\begin{abstract}
A B S T R A C T
In this Note we show that under suitable conditions on the data we can construct a sequence of solutions of the stochastic second grade fluid that converges to the probabilistic strong solution of the stochastic Navier-Stokes equations when the stress modulus $\alpha$ tends to zero.
\end{abstract}

(C) 2010 Published by Elsevier Masson SAS on behalf of Académie des sciences.

\section{R É S U M É}

Dans cette Note nous montrons que, sous des hypothèses appropriées sur les données, on peut construire une suite de solutions fortes des équations stochastiques pour les fluides de grade deux qui convergent vers les solutions fortes probabilistes des équations stochastiques de Navier-Stokes quand le module de contrainte $\alpha$ tend vers zéro.

(c) 2010 Published by Elsevier Masson SAS on behalf of Académie des sciences.

\section{Introduction and main result}

Let $D=[0, L]^{2} \subset \mathbb{R}^{2}, L>0$, be a periodic square, $T>0$ a fixed time. We consider a complete probability space $(\Omega, \mathcal{F}, P)$ endowed with the filtration $\mathcal{F}^{t}, 0 \leqslant t \leqslant T$, which is the $\sigma$-field generated by a given $\mathbb{R}^{m}$-valued standard Wiener process $\{\mathcal{W}(s), 0 \leqslant s \leqslant T\}$ and the null sets of $\mathcal{F}$. In this Note we investigate the behavior of the probabilistic strong solution of the following problem:

$$
\left\{\begin{array}{l}
\mathrm{d}\left(u^{\alpha}-\alpha \Delta u^{\alpha}\right)+\left(-v \Delta u^{\alpha}+\operatorname{curl}\left(u^{\alpha}-\alpha \Delta u^{\alpha}\right) \times u^{\alpha}+\nabla \mathfrak{P}\right) \mathrm{d} t=F \mathrm{~d} t+G \mathrm{~d} \mathcal{W} \text { in } \Omega \times(0, T] \times D, \\
\operatorname{div} u^{\alpha}=0 \text { in } \Omega \times(0, T] \times D, \\
\int_{D} u^{\alpha} \mathrm{d} x=0 \text { in } \Omega \times(0, T], \\
u^{\alpha}(0)=u_{0} \text { in } \Omega \times D,
\end{array}\right.
$$

when $\alpha \rightarrow 0$. The system (1), which is to be understood in the sense of distributions, is the equation of motion for an incompressible second grade fluid driven by random external forces. Here $u^{\alpha}$ is the velocity of the fluid, $\mathfrak{P}$ is a modified pressure given by

E-mail addresses: paulrazafi@gmail.com (P.A. Razafimandimby), sango1767@gmail.com, mamadou.sango@up.ac.za (M. Sango). 


$$
\mathfrak{P}=-\tilde{p}-(1 / 2)\left|u^{\alpha}\right|_{\mathbb{R}^{2}}^{2}+\alpha u^{\alpha} \cdot \Delta u^{\alpha}+(\alpha / 4) \operatorname{tr}\left(\nabla u^{\alpha}+\left(\nabla u^{\alpha}\right)^{t}\right) .
$$

Throughout we assume that (1) is subject to the periodic boundary condition. We refer to [10] and [7] for further reading on fluid of complexity two and on second grade fluids. These fluids are non-Newtonian and modelling a large class of dilute polymeric solutions, industrial fluids, slurry flows and food rheology. In the deterministic case, i.e. when $G(t, x) \equiv 0$, existence and uniqueness results are given in [6,5] for instance. It is known from [8] that under general assumption on the data the weak solution (in the partial differential equations sense) of second grade fluids equations converges weakly to the weak solution of the Navier-Stokes equations. We also refer to [3] for interesting discussions related to their relationship with other fluid models. Corresponding results for the stochastic case have not been established yet. The existence and uniqueness results of probabilistic strong solution have only recently been proved in [12]. In this Note, we show that we can construct a sequence $u^{\alpha_{j}}$ of stochastic strong solutions of (1) which converges in a certain sense (see Theorem 1.1 and Remark 1.2) to the stochastic strong solution of the stochastic Navier-Stokes equations (SNSE) as $\alpha_{j} \rightarrow 0$.

Throughout this Note we set $\mathbb{X}=X \times X$ for any Banach space $X$. The set of all periodic divergence free and infinitely differentiable functions with zero space average in $D$ is denoted by $\mathcal{V}$, and we set $\mathbb{V}$ (resp. $\mathbb{H}$ ) its closure in $\mathbb{H}^{1}(D)$ (resp. in $\mathbb{L}^{2}$ ). We endow $\mathbb{H}$ with the $\mathbb{L}^{2}$-scalar product denoted by $(.$, .). The space $\mathbb{V}$ is a Hilbert space with the gradient scalar product $((.,)$.$) which is equivalent to the usual \mathbb{H}^{1}(D)$-scalar product and the scalar product

$$
(u, v)_{\mathbb{V}}=(u, v)+\alpha((u, v)) .
$$

For any Banach space $X, p, r \geqslant 1$, we set $L^{p, r}(0, T, \Omega, X)=L^{p}\left(\Omega, \mathcal{F}, P ; L^{r}(0, T ; X)\right)$; see [11] or [13] for the definitions of these spaces. We denote by $C$ any unessential positive constant independent of $\alpha$, which may change from one line to the next.

We assume that

(I) $F=F(t, x)$ is a $\mathbb{V}$-valued function defined on $[0, T] \times D$ such that $\int_{0}^{T}|F(t, x)|_{\mathbb{V}}^{p}<\infty$, for any $2 \leqslant p<\infty$.

(II) $G=G(t, x)$ is a $\mathbb{V}^{\otimes m}$-valued function defined on $[0, T] \times D$ such that $\int_{0}^{T}|G(t, x)|_{\mathbb{V} \otimes m}^{p}<\infty$, for any $2 \leqslant p<\infty$.

(III) We also assume that $u_{0} \subset \mathbb{V} \cap \mathbb{H}^{3}$ is non-random and that there exists a positive constant $C$ independent of $\alpha$ such that $\left|u_{0}\right|_{\mathbb{V}}<C$. Suppose also that $v>0$.

Under the assumptions (I), (II) and (III) it was proved in [12] that for the prescribed stochastic basis $\left(\Omega, \mathcal{F}, P, \mathcal{F}^{t}\right)$ there exists an unique $\mathcal{F}^{t}$-adapted process $u^{\alpha}$ such that (1) holds $P$-a.s. in the sense of distributions. The process $u^{\alpha}$ satisfies

$$
E \sup _{0 \leqslant t \leqslant T}\left|u^{\alpha}\right|_{\mathbb{V}}^{p}+E \sup _{0 \leqslant t \leqslant T}\left|\operatorname{curl}\left(u^{\alpha}-\alpha \Delta u^{\alpha}\right)\right|^{p}<\infty, \quad \forall 2 \leqslant p<\infty .
$$

Moreover, almost surely the paths of the solution are $\mathbb{V}$-valued weakly continuous.

The main result of this Note is the following:

Theorem 1.1. Under the hypotheses (I)-(III) there exist a probability space $(\bar{\Omega}, \overline{\mathcal{F}}, \bar{P})$, a family of probability measures $\left(\Pi^{\alpha_{j}}\right), a$ probability measure $\Pi$ and stochastic processes $\left(\mathcal{W}^{\alpha_{j}}, u^{\alpha_{j}}\right),(\overline{\mathcal{W}}, v)$ such that the law of $\left(\mathcal{W}^{\alpha_{j}}, u^{\alpha_{j}}\right)($ resp. $(\overline{\mathcal{W}}, v))$ is $\Pi^{\alpha_{j}}($ resp. $\Pi)$ and $\mathcal{W}^{\alpha_{j}} \rightarrow \overline{\mathcal{W}}$ uniformly $\bar{P}$-a.s. when $j \rightarrow \infty\left(\alpha_{j} \rightarrow 0\right)$. The pair $\left(\mathcal{W}^{\alpha_{j}}, u^{\alpha_{j}}\right)$ satisfies $\bar{P}$-a.s. (1) in the sense of distributions and as $j \rightarrow \infty\left(\alpha_{j} \rightarrow 0\right)$

$$
\begin{aligned}
& u^{\alpha_{j}} \rightarrow v, \text { weakly in } L^{p}\left(\bar{\Omega}, \overline{\mathcal{F}}, \bar{P} ; L^{2}(0, T ; \mathbb{V})\right), \\
& u^{\alpha_{j}} \rightarrow v \text { weakly-* in } L^{p}\left(\bar{\Omega}, \overline{\mathcal{F}}, \bar{P} ; L^{\infty}(0, T ; \mathbb{H})\right),
\end{aligned}
$$

for $2 \leqslant p<\infty$, and $(\bar{\Omega}, \overline{\mathcal{F}}, \bar{P}, v, \overline{\mathcal{W}})$ is a solution of the SNSE:

$$
\left\{\begin{array}{l}
\mathrm{d} v+(-v \Delta v+(v \cdot \nabla v)+\nabla \mathcal{P}) \mathrm{d} t=F \mathrm{~d} t+G \mathrm{~d} \overline{\mathcal{W}} \text { in } \bar{\Omega} \times(0, T] \times D, \\
\operatorname{div} v=0 \text { in } \bar{\Omega} \times(0, T] \times D, \\
\int_{D} v \mathrm{~d} x=0 \text { in } \bar{\Omega} \times(0, T], \\
v(0)=u_{0} \text { in } \bar{\Omega} \times D .
\end{array}\right.
$$

Remark 1.2. Since we are in 2-D then it is known that under our hypotheses (I)-(III) the problem (5) has a probabilistic strong solution which is unique, see for example [9]. This implies that the process $v$ of the above theorem is a probabilistic strong solution of the stochastic Navier-Stokes equations (5).

We refer for instance to [1,2] for definition and existence results related to probabilistic weak solution of the SNSE. 


\section{Sketch of the proof}

We show that for $\alpha \in(0,1)$ we have:

$$
E \sup _{0 \leqslant s \leqslant T}\left(\left|u^{\alpha}(s)\right|^{2}+\alpha\left\|u^{\alpha}(s)\right\|^{2}\right)^{\frac{p}{2}}+E\left(\int_{0}^{T}\left\|u^{\alpha}(s)\right\|^{2} \mathrm{~d} s\right)^{\frac{p}{2}}<C, \quad \forall 2 \leqslant p<\infty .
$$

We also have that for any $\delta \in(0,1)$ :

$$
E \sup _{|\theta| \leqslant \delta} \int_{0}^{T-\delta}\left|u^{\alpha}(t+\theta)-u^{\alpha}(t)\right|_{\mathbb{H}^{-4}}^{2} \leqslant C \delta .
$$

Indeed by setting $\Phi=\mathbb{P}\left(u^{\alpha}-\alpha \Delta u^{\alpha}\right)$ ( $\mathbb{P}$ is the Leray projector), we show that

$$
\begin{aligned}
|\Phi(t+\theta)-\Phi(t)|_{\mathbb{H}^{-4}}^{2} \leqslant & C \theta \int_{t}^{t+\theta}\left\{v\left|\Delta u^{\alpha}\right|_{\mathbb{H}^{-4}}^{2}+\left|u^{\alpha} \cdot \nabla u^{\alpha}\right|_{\mathbb{H}^{-4}}^{2}+\alpha^{2} \sum_{j, k}\left|\partial_{j} \partial_{k}\left(u_{j}^{\alpha} \partial_{k} u^{\alpha}\right)\right|_{\mathbb{H}^{-4}}^{2}\right\} \mathrm{d} t \\
& +C \theta \int_{t}^{t+\theta}\left\{\alpha^{2} \sum_{j, k}\left[\left|\partial_{j}\left(\partial_{k} u_{j}^{\alpha} \partial_{k} u^{\alpha}\right)\right|_{\mathbb{H}^{-4}}^{2}+\left|\partial_{k}\left(\partial_{k} u_{j}^{\alpha} \nabla u_{j}^{\alpha}\right)\right|_{\mathbb{H}^{-4}}^{2}\right]+|F|^{2}\right\} \mathrm{d} t+2\left|\int_{t}^{t+\theta} G \mathrm{~d} \mathcal{W}\right|_{\mathbb{H}^{-4}}^{2} .
\end{aligned}
$$

Thanks to Sobolev product formulas (see for example [4]) we have

$$
\begin{aligned}
& \left|u^{\alpha} \cdot \nabla u^{\alpha}\right|_{\mathbb{H}^{-4}}^{2} \leqslant C\left|u^{\alpha}\right|^{2}\left|\nabla u^{\alpha}\right|^{2}, \\
& \alpha^{2}\left|\partial_{j} \partial_{k}\left(u_{j}^{\alpha} \partial_{k} u^{\alpha}\right)\right|_{\mathbb{H}^{-4}}^{2} \leqslant C \alpha^{2}\left|u^{\alpha}\right|^{2}\left|\nabla u^{\alpha}\right|^{2}, \\
& \alpha^{2}\left|\partial_{j}\left(\partial_{k} u_{j}^{\alpha} \partial_{k} u^{\alpha}\right)\right|_{\mathbb{H}^{-4}}^{2} \leqslant \alpha C \alpha\left|\nabla u^{\alpha}\right|^{2}\left|\nabla u^{\alpha}\right|^{2}, \\
& \alpha^{2}\left|\partial_{k}\left(\partial_{k} u_{j}^{\alpha} \nabla u_{j}^{\alpha}\right)\right|_{\mathbb{H}^{-4}}^{2} \leqslant \alpha C \alpha\left|\nabla u^{\alpha}\right|^{2}\left|\nabla u^{\alpha}\right|^{2} .
\end{aligned}
$$

With these estimates along with the Martingale inequality, the estimate (6) and the assumption on $G$, we deduce from (8) that

$$
E \int_{0}^{T-\delta} \sup _{0 \leqslant \theta \leqslant \delta}|\Phi(t+\theta)-\Phi(t)|_{\mathbb{H}^{-4}}^{2} \mathrm{~d} t \leqslant C \delta
$$

This implies that (7) holds for $\theta \geqslant 0$ (the case $\theta<0$ also holds).

Now, we introduce the mapping $\Phi: \omega \mapsto\left(\mathcal{W}(\omega), u^{\alpha}(\omega,).\right)$. The family of probability measures $\left(\Pi^{\alpha}\right)$ is defined on $\mathfrak{S}=$ $C\left(0, T ; \mathbb{R}^{m}\right) \times L^{2}(0, T ; \mathbb{H})$ by $\Pi^{\alpha}(S)=P\left(\Phi^{-1}(S)\right)$, for any $S \in \mathcal{B}(\mathfrak{S})(\mathcal{B}(\mathfrak{S})$ is the Borel $\sigma$-algebra of $\mathfrak{S})$. The family $\left\{\Pi^{\alpha}: 0<\right.$ $\alpha<1\}$ is tight. Thus by Prokhorov's theorem we can extract from $\left(\Pi^{\alpha}\right)$ a subsequence $\left(\Pi^{\alpha_{j}}\right)$, which weakly converges to a probability measure $\Pi$ on $\mathfrak{S}$. Skorokhod's embedding theorem ensures the existence of a complete probability space $(\bar{\Omega}, \overline{\mathcal{F}}, \bar{P})$ and random variables $\left(\mathcal{W}^{\alpha_{j}}, u^{\alpha_{j}}\right)$ and $(\overline{\mathcal{W}}, v)$ defined on $(\bar{\Omega}, \overline{\mathcal{F}}, \bar{P})$ with values in $\mathfrak{S}$ such that the probability law of $\left(\mathcal{W}^{\alpha_{j}}, u^{\alpha_{j}}\right)$ (resp. $\left.(\overline{\mathcal{W}}, v)\right)$ is $\Pi^{\alpha_{j}}$ (resp. $\left.\Pi\right)$ and

$$
\mathcal{W}^{\alpha_{j}}\left(\text { resp. } u^{\alpha_{j}}\right) \rightarrow \overline{\mathcal{W}}(\text { resp. } v) \text { in } C\left(0, T ; \mathbb{R}^{m}\right)\left(\text { resp. } L^{2}(0, T ; \mathbb{H}) \bar{P}\right. \text {-a.s. }
$$

Moreover, letting $\overline{\mathcal{F}}^{t}$ be the $\sigma$-algebra generated by $(\overline{\mathcal{W}}(s), v(s)), 0 \leqslant s \leqslant t$ and the null sets of $\overline{\mathcal{F}}$, we can show that $\overline{\mathcal{W}}$ is an $\overline{\mathcal{F}}^{t}$-adapted standard $\mathbb{R}^{m}$-valued Wiener process. We show that for any $j \geqslant 1, \phi \in \mathcal{V}$, for all $t \in[0, T]$, the following holds $\bar{P}$-a.s. (see [11])

$$
\left(u^{\alpha_{j}}, \phi\right)_{\mathbb{V}}+\int_{0}^{t}\left\{\left(v A u^{\alpha_{j}}+B\left(u^{\alpha_{j}}, u^{\alpha_{j}}\right), \phi\right)\right\} \mathrm{d} t=\left(u_{0}, \phi\right)_{\mathbb{V}}+\int_{0}^{t}\left(R\left(u^{\alpha_{j}}\right)+F\left(u^{\alpha_{j}}\right), \phi\right) \mathrm{d} t+\int_{0}^{t}(G, \phi) \mathrm{d} \mathcal{W}^{\alpha_{j}},
$$

where $B\left(u^{\alpha_{j}}, u^{\alpha_{j}}\right)=\mathbb{P}\left(u^{\alpha_{j}} . \nabla u^{\alpha_{j}}\right)$ and

$$
R\left(u^{\alpha_{j}}\right)=\alpha \sum_{i, k} \mathbb{P}\left(\partial_{i} \partial_{k}\left(u_{i}^{\alpha_{j}} \partial_{k} u^{\alpha_{j}}\right)+\partial_{i}\left(\partial_{k} u_{i}^{\alpha_{j}} \partial_{k} u^{\alpha_{j}}\right)-\partial_{k}\left(\partial_{k} u_{i}^{\alpha_{j}} \nabla u_{i}^{\alpha_{j}}\right)\right)
$$


Since $u^{\alpha_{j}}$ satisfies (14) then $u^{\alpha_{j}}$ satisfies the estimate (6). Consequently, we can extract from $\left(u^{\alpha_{j}}\right)$ a subsequence denoted by the same symbol such that

$$
\begin{array}{ll}
u^{\alpha_{j}} \rightarrow v & \text { weak- } * \text { in } L^{2}\left(\bar{\Omega}, \overline{\mathcal{F}}, \bar{P} ; L^{\infty}(0, T ; \mathbb{H})\right), \\
u^{\alpha_{j}} \rightarrow v & \text { weakly in } L^{2}\left(\bar{\Omega}, \overline{\mathcal{F}}, \bar{P} ; L^{2}(0, T ; \mathbb{V})\right) .
\end{array}
$$

We derive from (13), the estimate (6) and Vitali's Theorem that

$$
u^{\alpha_{j}} \rightarrow v \quad \text { strongly in } L^{2}\left(\bar{\Omega}, \overline{\mathcal{F}}, \bar{P} ; L^{2}(0, T ; \mathbb{H})\right) .
$$

Because of (9)-(12) and (6) we have

$$
\left(R\left(u^{\alpha_{j}}\right), \phi\right) \rightarrow 0 \quad \text { in } L^{2}\left(\bar{\Omega}, \overline{\mathcal{F}}, \bar{P} ; L^{2}(0, T)\right), \quad \forall \phi \in \mathcal{V} .
$$

Thanks to (16) we show that

$$
-\sum_{i, k} \bar{E} \int_{D \times[0, T]} u_{i}^{\alpha_{j}} \partial_{i} \phi_{k} \zeta u_{k}^{\alpha_{j}} \mathrm{~d} x \otimes \mathrm{d} t \rightarrow-\sum_{i, k} \bar{E} \int_{D \times[0, T]} v_{i} \partial_{i} \phi_{k} \zeta v_{k} \mathrm{~d} x \otimes \mathrm{d} t=\bar{E} \int_{0}^{T}\langle B(v, v), \zeta \phi\rangle \mathrm{d} t,
$$

for any $\zeta \in L^{\infty}(\bar{\Omega} \times[0, T], \mathrm{d} \bar{P} \otimes \mathrm{d} t)$ and $\phi \in \mathcal{V}$. That is

$$
\left\langle u^{\alpha_{j}} \cdot \nabla u^{\alpha_{j}}, \phi\right\rangle \rightarrow\langle v \cdot \nabla v, \phi\rangle \quad \text { weakly in } L^{2}\left(\bar{\Omega}, \overline{\mathcal{F}}, \bar{P} ; L^{2}(0, T)\right) \text { for any } \phi \in \mathcal{V} .
$$

We readily have

$$
\int_{0}^{t}(G, \phi) \mathrm{d} \mathcal{W}^{\alpha_{j}}-\int_{0}^{t}(G, \phi) \mathrm{d} \overline{\mathcal{W}} \quad \text { weakly in } L^{2}\left(\bar{\Omega}, \overline{\mathcal{F}}, \bar{P} ; L^{2}(0, T)\right) \quad \text { for any } \phi \in \mathcal{V} .
$$

It follows from (6) and (16) that

$$
\left(u^{\alpha_{j}}-\alpha_{j} \Delta u^{\alpha_{j}}-v, \phi\right) \rightarrow 0 \text { strongly in } L^{2}\left(\bar{\Omega}, \overline{\mathcal{F}}, \bar{P} ; L^{2}(0, T)\right) \text { for any } \phi \in \mathcal{V} .
$$

Using all these convergences we can derive from (14) that the following holds almost surely

$$
(v, \phi)+v \int_{0}^{t}\{((v, \phi))+(\mathbb{P}(v \cdot \nabla v), \phi)\} \mathrm{d} s=\left(u_{0}, \phi\right)+\int_{0}^{t}(F, \phi) \mathrm{d} s+\int_{0}^{t}(G, \phi) \mathrm{d} \overline{\mathcal{W}},
$$

for any $\phi \in \mathcal{V}$ and $t \in[0, T]$.

\section{Acknowledgement}

The authors acknowledge the support of the National Research Foundation of South Africa through a Focus Area grant.

\section{References}

[1] A. Bensoussan, Stochastic Navier-Stokes equations, Acta Applicandae Mathematicae 38 (1995) 267-304.

[2] A. Bensoussan, R. Temam, Equations Stochastiques du Type Navier-Stokes, Journal of Functional Analysis 13 (1973) $195-222$.

[3] A.V. Busuioc, On second grade fluids with vanishing viscosity, C. R. Acad. Sci. Paris, Ser. I 328 (12) (1999) $1241-1246$.

[4] J.-Y. Chemin, Perfect Incompressible Fluids, Clarendon-Oxford University Press.

[5] D. Cioranescu, V. Girault, Weak and classical solutions of a family of second grade fluids, Int. J. Non-Linear Mechanics 32 (2) (1997) 317-335.

[6] D. Cioranescu, E.H. Ouazar, Existence and uniqueness for fluids of second grade, in: Nonlinear Partial Differential Equations, College de France Seminar Pitman 109 (1984) 178-197.

[7] J.E. Dunn, R.L. Fosdick, Thermodynamics, stability and boundedness of fluids of complexity two and fluids of second grade, Arch. Rat. Mech. Anal. 56 (3) (1974) 191-252.

[8] D. Iftimie, Remarques sur la limite $\alpha \rightarrow 0$ pour les fluides de grade 2, C. R. Acad. Sci. Paris, Ser. I 334 (1) (2002) $83-86$.

[9] J.-L. Menaldi, S.S. Sritharan, Stochastic 2-D Navier-Stokes equations, Appl. Math. Optim. 46 (2002) 31-53.

[10] W. Noll, C. Truesdell, The Nonlinear Field Theory of Mechanics, Handbuch der Physik, vol. III, Springer-Verlag, Berlin, 1975.

[11] P.A. Razafimandimby, M. Sango, Weak solutions of a stochastic model for two-dimensional second grade fluids, Boundary Value Problems, vol. 2010, Article ID 636140, p. 47.

[12] P.A. Razafimandimby, M. Sango, Strong solution for a stochastic model of two-dimensional second grade fluids: Existence, uniqueness and stability, Preprint: http://users.aims.ac.za/ paul/publications.html.

[13] M. Sango, Magnetohydrodynamic turbulent flows: Existence results, Physica D: Nonlinear Phenomena 239 (12) (2010) $912-923$. 